\title{
DIVISION IX / COMMISSION 41 / WORKING GROUP HISTORICAL INSTRUMENTS
}

\author{
CHAIR \\ VICE-CHAIR \\ PAST CHAIR \\ BOARD MEMBERS
}

\author{
Sara J. Schechner \\ Tsuko Nakamura \\ Luisa Pigatto \\ Juergen Hamel \\ Kevin Johnson \\ Rajesh Kochhar \\ Nha Il-Seong \\ Wayne Orchiston \\ Bjørn Ragnvaid Pettersen \\ Shi Yunli
}

\section{TRIENNIAL REPORT 2009-2012}

\section{Introduction}

The Working Group on Historical Instruments (WG-HI) was founded by the members of Commission 41 at the 2000 Manchester IAU General Assembly with two main objectives: to assemble a bibliography of existing publications relating to historical instruments, and to encourage colleagues to carry out research and publish their results. Since then the concerns of the Working Group have expanded to include efforts to preserve and protect old astronomical instruments, observatories, and related sites as world cultural heritage and material evidence of the development of astronomy in different parts of the globe.

The WG-HI maintains liaisons with sister organizations through the involvement of its officers and board members in them. These include the Scientific Instrument Commission of the International Union for the History and Philosophy of Science / Division of History of Science and Technology (IUHPS-Scientific Instrument Commission); and the American Astronomical Society (AAS) Working Group for the Preservation of Astronomical Heritage.

\section{International Year of Astronomy (IYA) 2009 Initiative}

In 2007, the WG-HI of IAU Commission 41 (History of Astronomy) began to organize an interdisciplinary conference - "Astronomy and Its Instruments before and after Galileo" - to be held in Venice in 2009 on the 400th anniversary of Galileo's first observations with a telescope. The goals were expressed as follows:

"The conference aims to highlight mankind's path towards an improved knowledge of the sky using mathematical and mechanical tools as well as monuments and buildings, giving rise, in so doing, to scientific astronomy. It will analyze similarities and differences among cultures and countries in exploiting the shared resource that the sky represents, and will examine the historical-political and scientific background favoring the progress 
of scientific astronomy in different epochs and countries, progress that led to a crucial turning-point for observational astronomy when Galileo turned the telescope to the night sky and initiated the New Astronomy. A major aim of the meeting is to help move forward the process of ensuring the recognition and protection of cultural properties around the globe that bear powerful witness to the development of astronomy in diverse cultural contexts."

The plan was endorsed by Commission 46 (Astronomy Education and Development), Commission 55 (Communicating Astronomy with the Public), and by IAU Division XII (Union-Wide Activities).

Promoted as a joint symposium of the IAU and the INAF-Astronomical Observatory of Padova, "Astronomy and Its Instruments before and after Galileo" was held in VeniceSan Servolo Isle on 27 September - 3 October 2009. It was listed as an official event of UNESCO's International Year of Astronomy 2009, and sponsored by the IUHPS Scientific Instrument Commission. Patrons included UNESCO; the Istituto Nazionale di Astrofisica (INAF); the Università degli Studi di Padova, Italy; the Facoltà di Scienze, Matematiche, Fisiche e Naturali, Università di Padova; the Centro Interdipartimentale di Ricerca in Storia e Filosofia delle Scienze (CIRSFIS); the Centro per la Storia dell'Università di Padova; the Accademia Galileiana di Scienze, Lettere ed Arti in Padova; the Arab Union for Astronomy and Space Sciences (AUASS); the Società Astronomica Italiana (SAIt); the Comune di Venezia; the Provincia di Venezia; and the Regione del Veneto.

The conference program and other details can be found on the website: http://web.oapd.inaf.it/venice2009/index.php. The proceedings were published: Pigatto \& Zanini (2010).

\section{Conferences}

In addition to the aforementioned conference, several WG-HI members presented papers on astronomical instruments at the International Conference on Oriental Astronomy (ICOA-7), which was held at Mitaka, Tokyo in 2010. The proceedings are now in print: Nakamura, Orchiston, Sôma, \& Strom (2011).

Between 2009 and 2012, other Working Group members have taken part (or will take part) in meetings of the IUHPS Scientific Instrument Commission (Budapest, Florence, Kassel), the Historical Astronomy Division of the American Astronomical Society (Washington-DC, Seattle, Austin), the Antique Telescope Society (Ann Arbor, Charlottesville, Tuscon), and various special symposia featuring the history of the telescope. Many of these papers are in press.

Members of the Working Group are currently planning sessions on instruments for the General Assembly in Beijing in 2012. Among these, one session will focus on field expeditions, covering not only Transits of Venus (of particular interest in 2012), but also eclipses, determination of longitude, and so forth.

\section{Projects}

Prior to this triennium, the WG-HI had begun preparation of a thesaurus of historical instruments used in astronomy and related disciplines such as geography, geodesy, 
navigation, meteorology, and chronology. It was to be a list of terms plus variants and synonyms from different countries, etymologies, general definitions, related bibliographic sources, and images. A preliminary list of instruments was circulated among Working Group members. In 2009 during the current triennium, the project was discontinued when the Working Group learned that it duplicated work already done by the IUHPS Scientific Instrument Commission, museums with major holdings of historical scientific instruments, and other learned societies at the intersection of the history of astronomy and early scientific instruments. Moreover, the availability of these resources on the Web made the publication of a thesaurus by the WG-HI redundant.

In anticipation of the 2012 Transit of Venus, the WG-HI is encouraging knowledgeable scholars and museums holding apparatus used for past transits to collaborate by adding additional material to a Transit of Venus website created in 2004 by the IUHPS Scientific Instrument Commission (http://transits.mhs.ox.ac.uk).

Sara J. Schechner Chair of Working Group

\section{References}

Nakamura, T., Orchiston, W., Sôma, M., \& Strom, R. (eds.) 2011. Proceedings of the Seventh International Conference on Oriental Astronomy. Tokyo, National Astronomical Observatory of Japan.

Pigatto, L., \& Zanini, V. (eds.) 2010. Astronomy and Its Instruments before and after Galileo. Padua, Cooperativa Libraria Editrice Università di Padova. pp. 512. 